
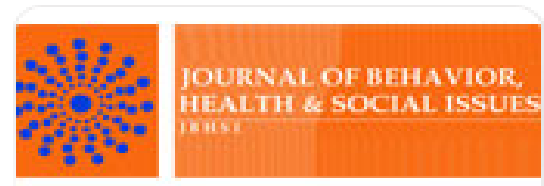

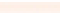

Journal of Behavior, Health \& Social Issues

ISSN: 2007-0780

jcpedro@unam.mx

Asociación Mexicana de Comportamiento y

Salud, A. C.

México

Rivera-Ledesma, Armando; Montero-López Lena, María; Sandoval-Ávila, Rosalba Escala de Ansiedad ante la Muerte, de Templer: propiedades psicométricas en pacientes con insuficiencia renal crónica terminal Journal of Behavior, Health \& Social Issues, vol. 2, núm. 2, noviembre-abril, 2010, pp. 83-91 Asociación Mexicana de Comportamiento y Salud, A. C.

Distrito Federal, México

Disponible en: http://www.redalyc.org/articulo.oa?id=282221720008

Cómo citar el artículo

- Número completo

- Más información del artículo

Página de la revista en redalyc.org

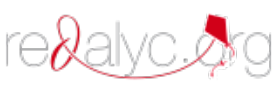

Sistema de Información Científica

Red de Revistas Científicas de América Latina, el Caribe, España y Portugal Proyecto académico sin fines de lucro, desarrollado bajo la iniciativa de acceso abierto 


\title{
ESCALA DE ANSIEDAD ANTE LA MUERTE, DE TEMPLER: PROPIEDADES PSICOMÉTRICAS EN PACIENTES CON INSUFICIENCIA RENAL CRÓNICA TERMINAL
}

\author{
TEMPLER'S DEATH ANXIETY SCALE: PSYCHOMETRIC PROPERTIES \\ IN TERMINAL CHRONIC RENAL FAILURE
}

\author{
Armando Rivera-Ledesma ${ }^{1}$ \\ María Montero-López Lena ${ }^{2}$ \\ Rosalba Sandoval-Ávila ${ }^{3}$ \\ 1 UNIVERSIDAD DE LONDRES, COORDINACIÓN DE \\ Investigación y Producción Científica. DistriIto \\ FEDERAL, MÉXICO. \\ 2 Universidad Nacional Autónoma de México, \\ Facultad de Psicología, División de Estudios de \\ Posgrado, Distritto Federal, México. \\ 3 Instituto Mexicano del Seguro Social. Hospital \\ General de Zona núm. 8. Jefatura de Trabajo \\ SOCIAl, DistriIto Federal, México.
}

\begin{abstract}
Los autores contribuyeron de manera semejante en todas las fases del escrito. Dirigir la correspondencia al primer autor, Universidad de Londres, Coordinación de Investigación y Producción Científica. Ciudad de México. Plaza de Luis Cabrera núm. 9, Col. Roma, 06700 Delegación Cuauhtémoc, México, D.F, correos electrónicos: armandoriveral@hotmail.com, mowilith@hotmail.com
\end{abstract}

RECIBIDO: 10 DE AGOSTO DE 2010

ACEPTADO: 27 DE OCTUBRE DE 2010

Resumen

En México la insuficiencia renal crónica (IRC) es un problema importante de salud pública con 4000 nuevos casos por año cuyo desenlace suele ser el tratamiento con diálisis peritoneal o hemodiálisis con una alta probabilidad pronóstica terminal. La presente investigación exploró la Escala de Ansiedad ante la Muerte, de Templer adaptada a sujetos mexicanos por Rivera-Ledesma y Montero-López Lena. Se empleó una muestra de 83 pacientes diabéticos con IRC terminal en tratamiento con diálisis peritoneal continua ambulatoria con una edad media de 53.5 años ( $\mathrm{DE}=15.3$ ); 71\% de la muestra fueron adultos mayores entre los 50 y los 82 años. Se evidenciaron dos factores que en conjunto explicaron $57.1 \%$ de la varianza con una consistencia interna alfa de .91, coincidiendo parcialmente con la estructura sugerida por la versión adaptada. Los dos factores y la escala total correlacionaron de manera importante y significativa entre sí sugiriendo homogeneidad en el constructo que determina el instrumento. Adicionalmente, la escala correlacionó significativamente con las variables depresión y ansiedad (entre .38 y .48, con $p<.01$ ), discriminando mayores grados de estas dos variables entre sujetos con baja y media ansiedad ante la muerte y aquellos con un alto grado de ésta $(F=$ 
Rivera-Ledesma, Montero-López \& Sandoval-Ávila

5.967, $\mathrm{gl}=2, p=.004$, y $F=7.685, \mathrm{gl}=2, p=.001$ ). Así, es posible concluir que aquellos sujetos con alta ansiedad ante la muerte pueden representar un grupo caracterizado por depresión y ansiedad significativas. Adicionalmente, se encontraron diferencias respecto al género: las mujeres parecen poseer una media más alta de ansiedad ante la muerte (media $=26.9, S=10.5$ ) que los hombres (media $=22.8, \mathrm{DE}=7.0 ; t=2.04$, $\mathrm{gl}=73.8, p=.044)$. Es deseable promover la asistencia psicológica a pacientes con IRC terminal a fin de favorecer su adaptación a la condición crónica de su padecimiento.

Palabras clave: Ansiedad ante la muerte, depresión, ansiedad, diabetes, muerte.

\section{Abstract}

In Mexico, chronic renal failure (CRF) is a major public health problem with 4000 new cases per year where the outcome is usually the peritoneal dialysis or hemodialysis with high probability of terminal prognosis. This research explored Templer's Death Anxiety Scale adapted in Mexican subjects by Rivera-Ledesma and Montero-López Lena (2010). The sample consisted of 83 diabetic patients with terminal CRF treated with continuous ambulatory peritoneal dialysis with a mean age of 53.5 years ( $\mathrm{SD}=15.3$ ), $71 \%$ of the sample were Seniors between 50 and 82 years old. It showed two factors that together accounted for $57.1 \%$ of the variance with an internal consistency alpha of .91, partly coinciding with the structure suggested by the adapted version. The two factors and total scale correlated significantly to each other suggesting homogeneity in the construct that determines the instrument. Additionally, the scale correlated significantly with Depression and Anxiety (between .38 and .48, $\mathrm{p}<.01$ ), discriminating higher levels of these two variables between subjects with low and medium anxiety about death and those with a high degree of this anxiety $(\mathrm{F}=5967, \mathrm{df}=2, \mathrm{p}=.004$ and $\mathrm{F}=7685, \mathrm{df}=2, \mathrm{p}=.001)$. It is possible to conclude that those subjects with high death anxiety may represent a group characterized by depression and significant anxiety. In addition, differences about sex: Women seem to have a highest average death anxiety (Mean $=26.9, S=10.5)$ than men (Mean $=22.8, \mathrm{SD}=7.0, \mathrm{t}=2.04, \mathrm{df}=73.8, \mathrm{p}=.044)$. It is desirable to promote psychological assistance to patients with terminal CRF to facilitate their adaptation to the chronic condition.

Keywords: Death anxiety, depression, anxiety, diabetes, death.

El hombre vive tan sólo para no morir; de tal suerte que la expectativa de dejar de existir no pueda ser vista sino con ansiedad por bien que se entienda lo irremediable de su ocurrencia y la necesidad de su asunción. La muerte es siempre una separación obligada; separación de este mundo y separación del otro. Cómo bien sostenía Caruso (2005): “Toda separación amorosa es una aproximación a la muerte", y lo que nos liga a este mundo y a aquellos que lo habitan es en definitiva el amor, el apego. Quizá cuando todos (o al menos esa mayoría significativa que llena nuestra historia) se hayan ido, quizás entonces sea más fácil asumir que hay que seguirles.

Se ha sugerido (Grau et al., 2008) que la muerte no es un fenómeno instantáneo, sino un proceso biológico, psicosocial y cultural. La ansiedad ante la muerte ha sido definida (Templer, 1970; Limonero, 1997) como una reacción emocional producida por la percepción de señales de peligro o amena- zas reales o imaginarias a la propia existencia que pueden desencadenarse por estímulos ambientales, situacionales o de disposición relacionados con la muerte propia o ajena.

La insuficiencia renal crónica (IRC) es un síndrome caracterizado por el deterioro progresivo de la función renal consecuente a la destrucción de la masa nefronal a partir de diversos factores etiológicos, entre los que se encuentran la glomerulonefritis $(37 \%)$, pielonefritis $(11 \%)$, riñón poliquístico $(9 \%)$, neuropatía diabética (9\%), hipertensión (7.5\%) y nefropatía de analgésicos (2\%), principalmente. En fases avanzadas de la enfermedad, los tratamientos de elección suelen ser la diálisis peritoneal continua ambulatoria (DPCA), la hemodiálisis (HD), e incluso el trasplante renal (Salgado y Guillén, 1990). En México la IRC es un problema importante de salud pública con una incidencia de aproximadamente 4000 nuevos casos por año (Alonso, Sánchez, Va- 
Ansiedad a la muerte en pacientes con insuficiencia renal

Ilejo y Ramos, 2005), cuyas principales causas parecen ser la diabetes mellitus (DM) y la hipertensión arterial sistémica (HAS) de acuerdo con informes del Instituto Mexicano del Seguro Social (López, 1994). La diabetes es en sí misma una enfermedad crónica de amplias proporciones en México; entre 8 y $10 \%$ de la población mayor de 20 años padece la enfermedad, con una mayor proporción de casos en edades superiores a los 40 años (Secretaría de Salud, [SS], 2001).

Las enfermedades crónicas terminales (cáncer, insuficiencia renal, cirrosis hepática, SIDA, etc.) suelen exponer al individuo a su propia muerte. El desenlace es generalmente conocido y por lo general suele llegar el momento en que el paciente debe ser manejado con cuidados paliativos. En realidad, nadie conoce el día en que el paciente cederá la vida, aunque la mayoría dé por sentado que en efecto morirá. Con todo, parece ser que un mejor estado psicológico respecto a la enfermedad terminal puede optimizar la calidad de vida del paciente, infundir una actitud más favorable y probablemente una mejor resistencia al deterioro físico y psicológico. Es ahí donde el sentido de vivir puede hacer el camino más transitable, como Frankl sostenía siguiendo a Nietzsche cuando afirmaba: "Quien tiene por qué vivir, puede aceptar casi cualquier cómo" (Grau et al., 2008).

En su trayectoria hacia la muerte, los enfermos terminales enfrentan múltiples temores (a la muerte en sí, a lo desconocido, al dolor, a perder a su familia y su identidad, etc.).

Se ha señalado (Grau et al., 2008) que, por consideraciones éticas, la mayor parte de las investigaciones realizadas en torno a la muerte se ha llevado a cabo en sujetos sanos que generalmente no se encuentran en peligro de perder la vida; Grau y colaboradores (2008) y Bayés, Limonero, Buendía, Burón y Enríquez (1999) han destacado un importante cuestionamiento: ¿hasta qué punto los datos así obtenidos pueden generalizarse a situaciones reales que suscitan una amenaza mortal? A fin de contribuir a la respuesta de esta interrogante, la presente investigación evaluó las propiedades psicométricas de la Escala de Ansiedad ante la Muerte, de Templer (1970) adaptada para adultos mayores mexicanos por Rivera-Ledesma y Montero-López Lena (2010), en una muestra de pacientes con insuficiencia renal crónica terminal en tratamiento con diálisis peritoneal continua ambulatoria.
Método

\section{Muestra}

La muestra fue de tipo no probabilístico, intencional. Se consideraron 83 pacientes diabéticos con insuficiencia renal crónica bajo tratamiento con diálisis peritoneal continua ambulatoria (DPCA), elegidos por disponibilidad de la Unidad de Diálisis de un hospital gubernamental en la Ciudad de México. Los criterios de inclusión fueron los siguientes: 1) sujetos alfabetos; 2 ) sin problemas de ceguera, sordera o mudez; 3) sin deterioro cognitivo evidente o grave; 4) estar en DPCA con antigüedad mínima de dos meses; 5) diabetes como causa documentada en expediente de la insuficiencia renal crónica; 6) que otorgaran su consentimiento informado para participar en la investigación.

La edad media del total de la muestra fue de 53.5 años (DE $=15.3$ ), con un rango de edad de 68 años (edad mínima: 14 , máxima: 82$) ; 71 \%(\mathrm{~N}=59)$ de la muestra estuvo compuesta por sujetos entre los 50 y los 82 años de edad (media $=61.6$ años, DE $=$ $7.4)$, y $29 \%$ del restante $(N=24)$ por sujetos entre los 14 y los 49 años (media = 33.5, DE = 10.8). Las mujeres representaron $51.9 \%(\mathrm{~N}=43)$ de la muestra; casi $82 \%(\mathrm{~N}=68)$ de los pacientes tenían pareja, y $76 \%(\mathrm{~N}=63)$ vivían con ella. El 80.7\% ( $\mathrm{N}=67)$ no trabajaban y el restante sí. Respecto al nivel educativo, la mayoría de los sujetos sólo tenían la primaria terminada (45.8\%, $\mathrm{N}=38$ ); el número de sujetos con secundaria, preparatoria y estudios de licenciatura o más, terminados fueron 22 (26.5\%), 14 (16.9\%) y $9(10.8 \%)$, respectivamente.

\section{Procedimiento}

El presente estudio forma parte de una investigación más amplia sobre DPCA, en la que los sujetos participantes fueron seleccionados por disponibilidad conforme acudían a la Unidad de diálisis de un hospital general gubernamental a una consulta de rutina ( $v . g r$., revisión de líquidos, consulta con el médico nefrólogo, suministro de medicamentos, etc.). A todos los sujetos se les explicó el objetivo de la investigación, respondiendo sus preguntas y resolviendo dudas. En los casos en que los pacientes aceptaron participar en la investigación, se les solicitó su consentimiento informado, y a continuación se les practicó una evaluación inicial a fin de explorar el cumplimiento de los criterios de inclusión. 
Rivera-Ledesma, Montero-López \& Sandoval-Ávila

Al efecto, se recabó información socio-demográfica mediante entrevista dirigida; se evaluó su estado cognitivo mediante el Mini-Mental State Examination de Folstein, Folstein y McHugh (1975) según la versión explorada por Ostrosky-Solis, López-Arango y Ardila (2000), y su estado funcional mediante la Escala de Actividades de la Vida Diaria (Katz, Ford, Moskowitz, Jackson, y Jaffee, 1963) y la Escala de Actividades Instrumentales de la Vida Diaria (Lawton, 1972; Lawton y Brody, 1969). Los pacientes con alteraciones cognitivas o con disfuncionalidad significativa (v.gr., problemas significativos de visión o audición, analfabetas), fueron descartados de la investigación; al efecto, se consideró un punto de corte menor o igual a 23 puntos. La muestra resultante obtuvo una media de 24.8, ( $\mathrm{DE}=3.8)$. Los pacientes con una funcionalidad conservada fueron seleccionados para la investigación; se les entregó un amplio cuestionario diseñado para ser contestado en su casa durante tres sesiones de aproximadamente cuarenta minutos cada una. Los instrumentos fueron recuperados en su mayoría a la semana siguiente. Una vez contestados los cuestionarios, la información fue capturada y analizada en el sistema PASW Statistics 18.

\section{Instrumentos}

Escala de Ansiedad ante la Muerte, EAAM (Templer, 1970; adaptada a sujetos mexicanos por Rivera-Ledesma y Montero-López Lena, 2010). En la versión adaptada a adultos mayores mexicanos, se evidenciaron tres factores (miedo a la muerte, miedo a la agonía o la enfermedad y miedo a que la vida llegue a su fin, que incluyeron 14 reactivos), los cuales explicaron $60.3 \%$ de la varianza con una consistencia interna alfa de .80. En la presente investigación, la escala mantuvo una consistencia interna alfa de .91.

Escala de Depresión del Centro de Estudios Epidemiológicos (Radloff, 1977, citado por Medina-Mora et al., 1992; Montero-López Lena, 1998). Con 20 reactivos, la escala mide sintomatología depresiva (afecto deprimido, sentimientos de culpa y minusvalía, sentimientos de desesperanza y desamparo, retardo psicomotor, pérdida del apetito y trastornos del sueño). Su consistencia interna reporta valores alfa entre .89 en adultos mayores y .93 en población general. En esta investigación fue de .92 .

Inventario de Ansiedad de Beck, IAB (Beck, Epstein, Brown \& Steer, 1988). El IAB es un inventario autoaplicado compuesto por 21 reactivos que describen diversos síntomas de ansiedad; se centra en los aspectos físicos relacionados con la ansiedad, evaluando su intensidad. Su consistencia interna ascendió a .95 en esta investigación por el método de Cronbach.

Escala de Actividades de la Vida Diaria, AVD. (Katz, Ford, Moskowitz, Jackson \& Jaffee, 1963). Evalúa capacidad funcional básica para bañarse, vestirse, asearse, moverse con independencia, continencia, alimentación). Su consistencia interna en esta investigación ascendió a .91 por el método de Cronbach.

Escala de Actividades Instrumentales de la Vida Diaria, AIVD. (Lawton, 1972; Lawton \& Brody, 1969). Evalúa la ejecución de actividades cotidianas que permiten vivir con independencia (uso del teléfono, realizar compras, preparar comidas, realizar tareas domésticas, utilizar medios de transporte, responsabilizarse de la toma de medicamentos, lavar ropa, manejar dinero). Su consistencia interna en esta investigación ascendió a .93.

Mini-Mental State Examination, MMSE (Folstein, Folstein \& McHugh, 1975; Ostrosky-Solis, LópezArango \& Ardila, 2000). Esta escala es una evaluación breve del estado cognitivo. Detecta deterioro cognitivo grave en sujetos con al menos cinco años de escolaridad (especificidad, 86.3\%; sensibilidad, $86.3 \%$ ). Su consistencia interna en esta investigación fue de .70 .

Resultados

Se analizó la estructura factorial de la escala por el método de componentes principales con rotación varimax y valores eigen mayores a 1. Los resultados obtenidos se describen en la tabla 1.

La estructura arrojó dos factores que en conjunto explicaron $57.1 \%$ de la varianza aparentemente vinculados a dos cuestiones: miedo a la muerte y miedo a la agonía y al fin de la vida. El primer factor fue similar al reportado para la versión adaptada en una muestra de sujetos sin enfermedades crónicas terminales; incluyó los mismos reactivos más el número ocho ("A menudo me preocupa lo rápido que pasa el tiempo") descartando el reactivo 11 ("Me asusta la posibilidad de sufrir un ataque al corazón"). El segundo factor estuvo compuesto por los reactivos restantes. 
Ansiedad a la muerte en pacientes con insuficiencia renal

Tabla 1.

Estructura factorial rotada de la Escala de Ansiedad ante la Muerte, de Templer en una muestra de pacientes con insuficiencia renal crónica terminal.

\begin{tabular}{lll}
\hline Reactivos & Miedo a la muerte & $\begin{array}{c}\text { Miedo a la agonía } \\
\text { y al fin de la vida }\end{array}$ \\
\hline 7. Molestia por pensamientos sobre la muerte. & .873 & \\
3. Nerviosismo si la gente habla de la muerte. & .837 & \\
1. Mucho miedo de morir. & .786 & \\
5. Miedo de morir. & .758 & \\
10. Preocupación por la otra vida. & .706 & \\
8. Preocupación por paso del tiempo. & .636 & \\
2. Pensamiento sobre la muerte. & .524 & .844 \\
13. Tercera guerra mundial. & & .787 \\
6. Miedo de tener cáncer. & & .666 \\
12. La vida es muy corta. & & .640 \\
11. Sufrir un ataque al corazón. & .524 & .627 \\
4. Pensar que tuvieran que operarme. & & .582 \\
9. Miedo de una muerte dolorosa. & & .575 \\
15. Motivos para temer el futuro. & $\mathbf{4 6 . 6 \%}$ & $\mathbf{1 0 . 5 \%}$ \\
14. Horror por ver un cadáver. & $\mathbf{. 8 9}$ & $\mathbf{. 8 6}$ \\
\hline Porcentaje de varianza explicada: & & \\
Alfa: &
\end{tabular}

Porcentaje de la varianza total explicada: $\mathbf{5 7 . 1 \%}$

Consistencia interna alfa total: .91

Nota: en esta tabla los reactivos se han descrito de manera abreviada. La versión completa de los mismos puede ser consultada en Rivera-Ledesma y Montero-López Lena (2010). Propiedades psicométricas de la Escala de Ansiedad ante la Muerte de Templer en sujetos mexicanos. Diversitas, 6, 1, 135-140

La consistencia interna para los dos factores descritos fue, de .89 para el factor de "Miedo a la muerte" y .86 para el de "Miedo a la agonía y al fin de la vida"; ambos fueron superiores a los obtenidos en la muestra de sujetos sin padecimientos crónicos terminales. En esa investigación el reactivo 14 ("Me horroriza ver un cadáver") fue removido siguiendo las recomendaciones de Nunnally y Bernstein (1995) respecto a la baja utilidad de emplear un sólo reactivo aislado en la evaluación de un atributo. En la presente investigación el reactivo 14 fue nuevamente descartado porque acusó una carga menor a .40 y no fue asignado a ninguno de los dos factores descritos. La consistencia interna alfa general fue de .91; más alta que la reportada con sujetos sin enfermedades crónicas terminales (alfa $=.83$ ).
Por otro lado, se analizó la relación entre los factores de la Escala de Ansiedad ante la Muerte y las variables depresión y ansiedad con los resultados descritos en la tabla 2.

Los resultados evidenciaron con claridad una fuerte asociación entre los factores de la EAAM y entre éstos y el total de la escala, lo cual da cuenta de la unidad conceptual del instrumento. Por otro lado, la importante asociación entre la EAAM con la depresión y la ansiedad parece coincidir con lo esperado en pacientes crónicos según es posible documentar en la bibliografía respectiva (Mercado, Delgadillo \& González, 2008; Lonetto \& Templer, 1988, citados por Tomer, 1997; Yauri, 2005; Hendon \& Epting, citados por Neimeyer, 2007; López \& Fernández, 2010; Pérez, 2006; López \& Calle, 2008). 
Rivera-Ledesma, Montero-López \& Sandoval-Ávila

Tabla 2.

Correlaciones bivariadas entre los factores de la Escala de Ansiedad ante la Muerte, de Templer y las variables depresión y ansiedad.

\begin{tabular}{lllll}
\hline & Miedo a la muerte & $\begin{array}{l}\text { Miedo a la agonía y } \\
\text { al fin de la vida }\end{array}$ & Depresión & Ansiedad \\
\hline $\begin{array}{l}\text { Miedo a la Muerte } \\
\begin{array}{l}\text { Miedo a la agonía y al fin de } \\
\text { la vida }\end{array}\end{array}$ & $\mathbf{. 6 7 ^ { * * }}$ & $.67^{* *}$ & $.38^{* *}$ & $.43^{* *}$ \\
\hline & 1 & $.40^{* *}$ & $.43^{* *}$ \\
Total EAAM & $.91^{* *}$ & $.92^{* *}$ & $.43^{* *}$ & $.48^{* *}$ \\
\hline
\end{tabular}

$\mathrm{EAAM}=$ Escala de Ansiedad ante la Muerte ${ }^{* *}$ Significativos con $p<.01$.

A fin de profundizar en estas relaciones se evaluó la capacidad de la EAAM para discriminar entre sujetos con distintos niveles de depresión y ansiedad. Al efecto se estimaron los valores normales de la distribución de puntuaciones totales de la escala, determinándose aquellos que correspondieron a un nivel bajo de ansiedad ante la muerte (los correspondientes a una puntuación Z menor o igual a -1); ansiedad ante la muerte media (puntuaciones entre valores $Z$ de $-1 \mathrm{a}+1$ ), y alta ansiedad ante la muerte (los correspondientes a una puntuación $Z$ mayor o igual a +1$)$. A continuación se practicó un análisis de varianza de una sola vía (ANOVA), decidiéndose para el análisis post hoc las pruebas de Tukey o de Games-Howel, según se determinará o no la existencia de homocedasticidad según la fórmula de Levene. Los resultados se describen en la tabla 3.De acuerdo con los resultados, la EAAM fue capaz de discriminar entre sujetos con bajo y alto nivel de ansiedad ante la muerte cuando ambos grupos son comparados en el grado de depresión o ansiedad que experimentan; no así entre aquellos con niveles bajo y medio de ansiedad ante la muerte. Así, es posible concluir que aquellos sujetos con alta ansiedad ante la muerte pueden representar un grupo caracterizado por depresión y ansiedad significativas.

El poseer un trabajo o carecer de él y el contar con una pareja o estar privado de ella no reportaron diferencias significativas respecto al grado de ansiedad ante la muerte. Al clasificar a los sujetos por grupos de edad (de 17 a 49; 50 a 59, 60 a 69 y de 70 a 82 años) tampoco se hallaron diferencias significativas. Sin embargo, sí se encontraron diferencias respecto al sexo: las mujeres parecen poseer una media más alta de ansiedad ante la muerte (media $=26.9, \mathrm{DE}$ $=10.5$ ) que los hombres (media $=22.8, \mathrm{DE}=7.0 ; t$ $=2.04, g l=73.8, p=.044)$.

Tabla 3.

Análisis de diferencias en depresión y ansiedad en tres grupos de ansiedad ante la muerte.

\begin{tabular}{|c|c|c|c|c|c|c|c|}
\hline Depresión & $\mathbf{N}$ & Media & $\mathbf{S}$ & ANOVA & Levene & Post hoc & $\begin{array}{l}\text { Subconjuntos } \\
\text { homogéneos }\end{array}$ \\
\hline Baja & 15 & 33.4 & 8.8 & \multirow{4}{*}{$\begin{array}{l}F=5.967 \\
g l=2 \\
p=.004\end{array}$} & \multirow{4}{*}{$p=.381$} & Tukey: & \multirow{4}{*}{$B=M, p=.916$} \\
\hline Media & 54 & 34.5 & 7.9 & & & $B \neq A, p=.011$ & \\
\hline Alta & 14 & 42.6 & 9.4 & & & $M \neq A, p=.005$ & \\
\hline Total & 83 & 35.7 & 8.8 & & & & \\
\hline \multicolumn{8}{|l|}{ Ansiedad } \\
\hline Baja & 15 & 30.6 & 8.3 & \multirow{4}{*}{$\begin{array}{l}\mathrm{F}=7.685 \\
g l=2 \\
p=.001\end{array}$} & \multirow{4}{*}{$p=.974$} & Tukey: & \multirow{4}{*}{$\mathrm{B}=\mathrm{M}, p=.553$} \\
\hline Media & 54 & 33.2 & 7.5 & & & $B \neq A, p=.001$ & \\
\hline Alta & 14 & 41.2 & 8.6 & & & $M \neq A, p=.003$ & \\
\hline Total & 83 & 34.1 & 8.4 & & & & \\
\hline
\end{tabular}

$\mathrm{B}=$ Baja, $\mathrm{M}=$ Media, $\mathrm{A}=$ Alta 
Ansiedad a la muerte en pacientes con insuficiencia renal

\section{Discusión}

El objetivo de la presente investigación fue evaluar las propiedades psicométricas de la Escala de Ansiedad ante la Muerte, de Templer (1970; ) adaptada para adultos mayores mexicanos por Rivera-Ledesma y Montero-López Lena (2010), en una muestra de pacientes con insuficiencia renal crónica terminal en tratamiento con diálisis peritoneal continua ambulatoria. Los resultados permitieron mostrar para la escala una estructura factorial que explicó $57.1 \%$ de la varianza con dos factores (miedo a la muerte y miedo a la agonía o fin de la vida), con una consistencia interna favorable (alfa $=.91)$. Estos resultados parecen mostrar la idoneidad de la EAAM para su uso con pacientes crónicos terminales. La escala mostró una amplia mejoría respecto a sus propiedades psicométricas; si bien la estructura se vio reducida a dos factores, estos lograron explicar 3.3\% más de la varianza con una mayor consistencia interna (de .83 a .91). En la versión adaptada en sujetos mexicanos sanos de la comunidad aportó tres factores con valores alfa de .83, .69 y .65, respectivamente; en pacientes con IRC la consistencia interna de los factores fue notablemente mejor (.89 y .86) para sus dos factores.

Por otro lado, las asociaciones halladas entre los factores de la EAAM y las variables depresión y ansiedad parecen ser consistentes con la literatura relacionada con muestras de pacientes crónicos. Lonetto y Templer (1986, citado por Neimeyer, 1997) afirman que la ansiedad ante la muerte está conformada por la anticipación de las alteraciones físicas, reales o imaginarias, asociadas a las enfermedades graves, a la percepción sobre la limitación del tiempo y por la preocupación por el dolor que puede acompañar a la enfermedad y a la muerte. Esto se ve reflejado en los niveles moderados y graves de depresión ante la muerte. La depresión cobra sentido en los pacientes crónicos, ya que su condición supone reacciones físicas y psicológicas que causan disminución de energía física y sufrimiento físico, lo cual produce efectos a nivel emocional generados por el temor a la soledad o la invalidez, sensación de amenaza, etc. (Yauri, 2005).

En la presente investigación, la asociación entre las sub-escalas de "Miedo a la muerte" y "Miedo a la agonía y al fin de la vida" fue alta y significativa. Al respecto, un estudio realizado por Pollack (1979) evidenció que el mayor de los temores expresado por los sujetos estaba relacionado más con el miedo al dolor, a las operaciones, a lo que ocurre con el cuerpo después de la muerte y a la descomposición del cuerpo, que con el miedo a la muerte en sí. Esta idea coincide con el trabajo de Bayés, Limonero, Barreto y Comás (1997), quienes resaltan la diferencia entre la muerte y el proceso de morir. Los investigadores observaron en un grupo de estudiantes que el aspecto que genera mayor ansiedad es el propio proceso de morir, y el que menos, la idea de la muerte en sí misma.

Por otro lado, las diferencias halladas entre hombres y mujeres respecto al grado en que cada uno experimenta la ansiedad ante la muerte fueron consistentes con lo reportado por distintos investigadores (v.gr., Neymeyer, Wittkowski \& Moser, 2004), aunque contrastantes con otros (Elizalde y Flores, 2009); al parecer las mujeres tienden a experimentar sentimientos más intensos de ansiedad ante la muerte.

Quedan aspectos que aún deben ser investigados y que han sido documentados en la bibliografía en sujetos de otras latitudes. Por ejemplo: Mercado, Delgadillo y González (2008) compararon pacientes ambulatorios contra pacientes hospitalizados, encontrando diferencias significativas en el grado de ansiedad ante la muerte entre ambos grupos, presumiblemente debidas a la idea concebida del hospital como un lugar seguro donde se recibe cuidado y alivio y, por lo tanto, menos dolor (Yauri, 2005; Hendon \& Epting, citado por Neimeyer, 2007). Otros estudios parecen poner en relieve la necesidad de evaluar no sólo la asociación de la ansiedad ante la muerte con variables clínicas pertinentes, sino de evaluar su prevalencia en comparación con grupos normativos de sujetos sanos.

Podría decirse que la enfermedad no es en sí lo que produce la ansiedad ante la muerte, sino el grado de deterioro físico que produce la enfermedad (Fortner \& Neimeyer, 1999; y Lucas, 1974).

Al parecer, la educación sobre la muerte y la formación en cuidados paliativos han puesto de manifiesto su eficacia en la reducción de los niveles de ansiedad ante la muerte, en lograr una actitud más positiva hacia los ancianos y enfermos terminales y en mejorar la eficiencia en el trato con el enfermo y su familia (Burney-Benfield, 1994). La educación es el motor de cambio de las sociedades; el resorte por el cual las actitudes pueden cambiar. Las ciencias 
Rivera-Ledesma, Montero-López \& Sandoval-Ávila

de la salud no pueden pasar por alto la muerte, ni deben sobrestimarla, ni concederle una importancia menor de la que tiene. Deben considerarse obviamente prioritarios tanto el estudio de las emociones que acompañan al proceso como la práctica del soporte emocional necesario para afrontarla (López \& Fernández, 2010).

\section{Referencias}

Alonso, M. L. A., Sánchez, V. P. M., Vallejo, G. J. L., \& Ramos, C. M. G. (2005). Competencia y productividad laboral de pacientes en control con diálisis automatizada, Boletín Salud en el Trabajo, IMSS, 8 (47), 1-2.

Bayés, R., Limonero, J. T., Buendía, B., Burón, E., \& Enríquez, N. (1999). Evaluación de la ansiedad ante la muerte. Medicina Paliativa, 6, 140-143.

Bayés, R., Limonero, J. T., Barreto, P., \& Comas, M. (1997) A way to screen for suffering in palliative care. Journal of Paliative Care, 13, 22-26.

Beck, A. T., Epstein, N., Brown, G., \& Steer, R. A. (1988). An inventory for measuring clinical anxiety: Psychometric properties. Journal of Consulting and Clinical Psychology, 56, 893-897, available via: http://dx.doi.org/10.1037/0022006X.56.6.893

Burney-Banfield, S. (1994). Preparing students for their patients' death. Australian Journal of Advanced Nursing, 11, pp. 24-28.

Caruso, I. (2005). La separación de los amantes. México: Siglo XXI Editores.

Elizalde, G. C., \& Flores, G. A. V. (2009). Escala de ansiedad ante la muerte para ancianos mexicanos. En R.A.L.M. González-Celis, (Ed.). Evaluación en psicogerontología. México: Manual Moderno.

Folstein, M.F., Folstein, S. E., \& McHugh, P. R. (1975). "Mini-mental state". A practical method for grading the cognitive state of patients for the clinician". Journal of psychiatric research 12 (3), 189-98, available via: http://dx.doi. org/10.1016/0022-3956(75)90026-6

Fortner, B. V., \& Neinmeyer, R. A. (1999). Death anxiety in older adults: a quantitative review. Death Studies, 23, 387-411, available via: http:// dx.doi.org/10.1080/074811899200920

Grau A. J., Llantá, A. M. C., Massip, P. C., Chacón R. M., Reyes, M. M. C., Infante, P. O., \& Mora- les, A. D. (2008). Ansiedad y actitudes ante la muerte: revisión y caracterización en un grupo heterogéneo de profesionales que se capacita en cuidados paliativos. Pensamiento Psicológico, 4,10, 27-58.

Katz, S. C. Ford, A. B. Moskowitz, R. W., Jackson, B.A., \& Jaffee, M. W. (1963). Studies of illness in the aged. The Index of ADL: A standardized measure of biological and psychosocial function. Journal of the American Medical Association. 185, 914-919.

Lawton, M. P. (1972). Assessing the competence of older people. En D. Kent, R. Kastenbaum y S. Sherwood (Eds.), Research Planning and Action for the elderly. Nueva York: Behavioral Publications.

Lawton, M. P., \& Brody, E. M. (1969). Assessment of older people: Self-mantaining and instrumental of daily living. The Gerontologist, 9, 179-185.

Limonero, J. T. (1997). Ansiedad ante la muerte. Ansiedad y Estrés, 3, 37-46.

Lonetto, H., \& Templer, D. I. (1986). Death Anxiety. Washington: Hemisphere Publishing.

López, (1994). La transición epidemiológica, Ciencia Médica, 1 (26), 11-17.

López, C. A., \& Calle, S.I. (2008). Características psicométricas de la Escala de Ansiedad ante la muerte (DAS) en pacientes de VIH/SIDA. Psicothema, $20(4$,$) 958-963.$

López, C. A., \& Fernández, E. L. (2010) Variables influyentes de la depresión ante la muerte (DDSR) en estudiantes universitarios, disponible vía: http://www.educacion.udc.es/grupos/gipdae/ congreso/VIIIcongreso/pdfs/463.pdf consultado el 29 de julio de 2010.

Lucas, R. A. (1974). A comparative study of measures of general anxiety and death anxiety among three medical groups including patient and wife. Omega: Journal of Death and Dying, 5, 233-243, available via: http://dx.doi.org/10.2190/0V21FPKG-AJUB-4E7L

Medina-Mora, M., Rascón, M. L., Tapia, R., Mariño, M. C., Juárez, F., Villatoro, J., \& Gómez, M. (1992). Trastornos emocionales en población urbana mexicana: resultados de un estudio nacional. Anales del Instituto Mexicano de Psiquiatría.

Mercado, M. A., Delgadillo G. L. G., \& González J. I. (2008). Ansiedad ante la muerte en pacientes 
Ansiedad a la muerte en pacientes con insuficiencia renal

hospitalizados y ambulatorios. Revista de Psicología de la UAEM, I (I), 5-27.

Montero y López-Lena, M., (1998). Soledad y Depresión: ¿Fenómenos equivalentes o diferentes?, La Psicología Social en México, AMEPSO, (VII), 62-67.

Neufeldt, D. E., \& Colmes, C.B. (1979). Relationship between Personality Traits and Fear of Death. Psychological Reports, 45, 907-910.

Neymeyer, R. (1997). Métodos de evaluación de la ansiedad ante la muerte, Paidós, Barcelona.

Neymeyer, R. A., Wittkowski, J. \& Moser, R. P. (2004). Psychological research on death attitudes: An overview and evaluation. Death Studies, 28 (4), 309-340, available via: http://dx.doi. org/10.1080/07481180490432324

Nunnally, J. C., \& Bernstein, I. J. (1995). Teoría Psicométrica. (3a. ed.) México: McGraw-Hill.

Ostrosky-Solís, F., López-Arango, G., \& Ardila, A. (2000). Sensitivity and Specificity of the Mini-Mental State Examination in a SpanishSpeaking Population. Applied Neuropsychology. 7 (1), 25-31, http://dx.doi.org/10.1207/ S15324826AN0701_4

Pérez, L. A. V. (2006). El proceso tanatológico en pacientes con cáncer en fase terminal y aquellos con posibilidades de curación. Revista de la Sociedad Española e Internacional de Tanatología, (8), 6-12.
Pollack, J. M. (1979). Correlates of death anxiety: a review of empirical studies. Omega: Journal of Death an Dying, 10, 97-121. Available via: http:// dx.doi.org/10.2190/4KG5-HBH0-NNME-DM58

Rivera-Ledesma, A., \& Montero-López, M. (2010). Propiedades psicométricas de la Escala de Ansiedad ante la Muerte, de Templer en sujetos mexicanos. Diversitas, 6 (1),135-140.

Salgado, A., \& Guillen, F. (1990). Manual de Geriatría, Salvat Editores, Barcelona, España.

SS, Secretaría de Salud (2001). Encuesta Nacional de Enfermedades Crónicas. Gobierno de México.

Templer D. I. (1970). The construction and validation of a Death Anxiety Scale. Journal of General Psychology, 82, 165-177, available via: http:// dx.doi.org/10.1080/00221309.1970.9920634

Tomás-Sábado, J., \& Gómez-Benito, J. (2003). Variables relacionadas con la ansiedad ante la muerte. Revista de Psicología General y Aplicada, 56 (3), 257-279.

Tomer, A. (1997), Las actitudes hacia la muerte en la vida adulta, Taylor y Francis, Filadelfia.

Yauri, R. (2005). Aspectos psicológicos del paciente frente a la enfermedad. http://www.ilustrados. com/publicaciones/EpypAApAyukWMoifWN. php consultado el 4 de agosto del 2010. 